neglected the temperature drop caused by the dissociation; so the correct value of $s$ would be considerably smaller. Further experimental work seems necessary before a more refined analysis would be worthwhile.

\section{CONCLUSION}

$\mathrm{CN}$ and $\mathrm{C}_{2}$ are commonly observed molecules with intense spectra in the visible region so that they should prove to be particularly suitable substances to use for nvestigations of the detailed questions of chemical kinetics such as the relative reactivities of individual vibrational and rotational states. It is hoped that this work has provided some information about the over-all kinetics of their reactions which will be a useful guide for later studies.

\section{ACKNOWLEDGMENT}

It is a pleasure to thank Dr. F. H. Mies for many profitable discussions and Dr. H. T. Knight for providing the calculations of equilibrium shock properties.

\title{
On the Optical Spectra of Some Transition Metal Pentacyanonitrosyl Complexes
}

\author{
Harry B. Gray* and C. J. Ballhausen \\ The Institute for Physical Chemistry, University of Copenhagen, Denmark
}

(Received August 24, 1961)

\begin{abstract}
The visible absorption spectra of the ions $\left[\mathrm{Fe}(\mathrm{CN})_{5} \mathrm{NO}^{2-},\left[\mathrm{Mn}(\mathrm{CN})_{5} \mathrm{NO}^{3-}\right.\right.$, and $\left[\mathrm{Cr}(\mathrm{CN})_{5} \mathrm{NO}^{3-}\right.$ are interpreted using a crystal field level scheme of $C_{4 v}$ symmetry. Assuming a strong axial compression to be present a self-consistent assignment is obtained for both the $3 d^{5}$ and $3 d^{6}$ electronic configurations.
\end{abstract}

$\mathbf{T}$ HE purpose of this note is to discuss possible assignments for the characteristic absorption bands found in the visible spectra of several transition metal pentacyanonitrosyls. In particular we shall confine our attention to the spectra of $\left[\mathrm{Fe}(\mathrm{CN})_{5} \mathrm{NO}^{2-}\right.$ and $\left[\mathrm{Mn}(\mathrm{CN})_{6} \mathrm{NO}^{3-}\right.$, measured by Cotten et al., ${ }^{1}$ and to $\left[\mathrm{Cr}(\mathrm{CN})_{6} \mathrm{NO}^{3-}\right.$, measured by Bernal and Harrison. ${ }^{2}$

These complex ions are pictured as derived from the corrsponding hexacyanides, by replacing a CN group with an $\mathrm{NO}$ group. Thus the symmetry is lowered from $0_{h}$, in the hexacyanides, to effectively $C_{4 v}$, in the pentacyanonitrosyls. In an octahedral field the degenerate $3 d$ orbitals of the free metal ion split up into two groups of orbitals of symmetries $t_{2 g}$ and $e_{g}$. When the symmetry is lowered from $0_{h}$ to $C_{4 v}$ the $t_{2 g}$ orbitals split up into $e$ and $b_{2}$ orbitals, while the $e_{g}$ orbitals split into an $a_{1}$ and a $b_{1}$. The energies of these orbitals are given by the following expressions ${ }^{3}$ :

$$
\begin{aligned}
& E\left(a_{1}\right)=\epsilon_{0}+6 D q-2 D s-6 D t, \\
& E\left(b_{1}\right)=\epsilon_{0}+6 D q+2 D s-D t, \\
& E\left(b_{2}\right)=\epsilon_{0}-4 D q+2 D s-D t, \\
& E(e)=\epsilon_{0}-4 D q-D s+4 D t .
\end{aligned}
$$

*National Science Foundation Postdoctoral Fellow, 1960-61. Present address: Department of Chemistry, Columbia University, New York 27, New York.

1 F. A. Cotton, R. R. Monchamp, R. J. M. Henry, and R. C. Young, J. Inorg. Nucl. Chem. 10, 28 (1959).

2 I. Bernal and S. E. Harrison, J. Chem. Phys. 34, 102 (1961).

${ }^{3}$ For example, see C. J. Ballhausen and W. Moffitt, J. Inorg. Nuclear Chem. 3, 178 (1956).
The parameters $D s$ and $D t$ specify the degrees and the nature of the distortion of the octahedral ions. The parameter $D t$ is directly related to a tetragonal distortion along the $z$ axis. Hence we take $D t<0$ for a strong axial destabilization of the $a_{1}$ orbital, and $D t>0$ for a perturbation resulting in a lowering of the $a_{1}$ orbital as compared to the unperturbed $e_{\sigma}$ level in $0_{h}$ symmetry. ${ }^{3}$ In addition, since the $a_{1}$ orbital is directed along the fourfold symmetry axis in $C_{4 v}$, it is higher or lower in energy with respect to $b_{1}$ depending on whether there is axial destabilization or stabilization. However, it is not possible to specify a priori the relative energies of the $e$ and $b_{2}$ orbitals for either axial compression or elongation, since this depends on the relative importance of the $D s$ and $D t$ terms for the case under consideration.

The isoelectronic ions $\left[\mathrm{Fe}(\mathrm{CN})_{5} \mathrm{NO}^{2-}\right.$ and $\left[\mathrm{Mn}(\mathrm{CN})_{5} \mathrm{NO}^{3-}\right.$ will be considered first. These complexes must be formulated as containing $\mathrm{NO}^{+}$and, respectively, $\mathrm{Fe}^{2+}$ and $\mathrm{Mn}^{+}$, on the basis of their magnetic properties and infrared spectra. Thus the electronic configuration on each metal ion is effectively $3 d^{6}$; in the tetragonal crystal field this configuration becomes $(e)^{4}\left(b_{2}\right)^{2}$, giving a ground state ${ }^{1} A_{1}$. This ground state is consistent with the observed diamagnetism of the complexes.

The visible spectra of aqueous solutions of $\mathrm{Na}_{2}\left[\mathrm{Fe}(\mathrm{CN})_{5} \mathrm{NO}\right] \cdot 2 \mathrm{H}_{2} \mathrm{O}$ and $\mathrm{K}_{3}\left[\mathrm{Mn}(\mathrm{CN})_{5} \mathrm{NO}\right]$ are rather similar, ${ }^{1}$ showing bands with maxima at 20080 $\mathrm{cm}^{-1}(\epsilon=8.9)$ and $25640 \mathrm{~cm}^{-1}(\epsilon=24.6)$ in the former, 
Table I. Lowest spin singlet states for the $3 d^{6}$ electronic configuration in a strong crystal field with $\mathrm{C}_{4 v}$ symmetry.

\begin{tabular}{cccc}
\hline $\begin{array}{c}\text { Electron } \\
\text { con- } \\
\text { figuration }\end{array}$ & $\begin{array}{c}\text { Designa- } \\
\text { tion }\end{array}$ & $\begin{array}{c}\text { Orbital } \\
\text { energy }\end{array}$ & $\begin{array}{c}\text { Slater- } \\
\text { Condon } \\
\text { energy }\left(+15 F_{0}\right)\end{array}$ \\
\hline$e^{4} b_{2}{ }^{2}$ & ${ }^{1} A_{1}$ & $6 \epsilon_{0}-24 D q+14 D t$ & $-30 F_{2}-60 F_{4}$ \\
$e^{4} b_{2} b_{1}$ & ${ }^{1} A_{2}$ & $6 \epsilon_{0}-14 D q+14 D t$ & $-30 F_{2}-95 F_{4}$ \\
$e^{4} b_{2} a_{1}$ & ${ }^{1} B_{2}$ & $6 \epsilon_{0}-14 D q-4 D s+9 D t$ & $-14 F_{2}-175 F_{4}$ \\
$e^{3} b_{2}{ }^{2} a_{1}$ & ${ }^{1} E(1)$ & $6 \epsilon_{0}-14 D q-D s+4 D t$ & $-26 F_{2}-115 F_{4}$ \\
$e^{3} b_{2}{ }^{2} b_{1}$ & ${ }^{1} E(2)$ & $6 \epsilon_{0}-14 D q+3 D s+9 D t$ & $-18 F_{2}-155 F_{4}$ \\
\hline
\end{tabular}

a Configuration interaction between ${ }^{1} E(1)$ and ${ }^{1} E(2)$ is neglected.

and $18520 \mathrm{~cm}^{-1}(\epsilon=23.6)$, and $28740 \mathrm{~cm}^{-1} \quad(\epsilon=114)$ in the latter. The excited states which can be obtained by moving one electron from the configuration $(e)^{4}\left(b_{2}\right)^{2}$ into the $b_{1}$ and $a_{1}$ orbitals are listed in Table I, along with their orbital and Slater-Condon energies. In calculating these energies we have assumed that the tetragonal distortion is more important than the electron correlation forces.

Barring unreasonably large values for $D s$ and $D t$, it is clear from a consideration of the Slater-Condon parameters that the states ${ }^{1} A_{2}$ and ${ }^{1} E(1)$ should be lowest in energy, and thus responsible for the two observed bands. More specifically, the first band is assigned to be transition ${ }^{1} A_{1} \rightarrow{ }^{1} A_{2}$ and the second band to the transition ${ }^{1} A_{1} \rightarrow^{1} E(1)$, for the following reasons: first, in $C_{4 v}$ symmetry with an $A_{1}$ ground state, only transitions to $A_{1}$ and $E$ states are orbitally allowed. Since the intensity of the second band is appreciably greater than the intensity of the first band, it is more likely to be the orbitally allowed ${ }^{1} A_{1} \rightarrow^{1} E(1)$ transition; second, there is reason to believe that a strong axial compression is present in the metal cyanonitrosyls. This belief is fostered by the fact that the $\mathrm{M}-\mathrm{N}$ bond lengths in first-row transition metal-nitric oxide complexes range from 1.57 to $1.77 \mathrm{~A},{ }^{4}$ while the $\mathrm{M}-\mathrm{C}$ bond lengths in corresponding metal cyanide complexes are about $1.9 \mathrm{~A} .5,6$ Such a short $\mathrm{M}-$-NO distance, if present in the metal cyanonitrosyls, would result in a strong axial destabilization $(D t<0)$, stabilizing the ${ }^{1} A_{2}$ excited state.

Griffith et $a l .^{7}$ have recently reported the preparation, infrared spectrum, and magnetic susceptibility of $\mathrm{K}_{3}\left[\mathrm{Cr}(\mathrm{CN})_{5} \mathrm{NO}\right] \cdot \mathrm{H}_{2} \mathrm{O}$. The compound has one unpaired electron, and was formulated as containing $\mathrm{Cr}^{+}$, with the electronic configuration $3 d^{5}$, and $\mathrm{NO}^{+}$mainly on the basis of infrared evidence. Another possibility is $\mathrm{Cr}^{3+}\left(3 d^{3}\right)$ with $\mathrm{NO}^{-}$, but a simple calculation shows that in order to pair two electrons in $\mathrm{Cr}^{3+}$, to obtain

4 J. Lewis, Sci. Progr. 47, No. 187, 506 (1959).

${ }^{6}$ V. A. Pospelov and G. S. Zhdanov, J. Phys. Chem. (U.S.S.R.) 21, 405 (1947).

${ }^{6}$ D. T. Cromer, J. Phys. Chem. 61, 1388 (1957).

7 W. P. Griffith, J. Lewis, and G. Wilkinson, J. Chem. Soc. 1959, 872 . either configuration $(e)^{3}$ or $\left(b_{2}\right)^{2}(e)$ [see Eqs. (1)-(4)], the separation between the $e$ and $b_{2}$ orbital levels $(3 D s-5 D t)$ would have to be approximately 20000 $\mathrm{cm}^{-1}$. Since a separation of this magnitude is unlikely, and further, since the measured ${ }^{2} g$ factor of 1.995 is inconsistent with either of these configurations, the formulation as $\mathrm{Cr}^{+}$and $\mathrm{NO}^{+}$appears to be the only acceptable possibility.

The optical spectrum of crystalline $\mathrm{K}_{3}\left[\mathrm{Cr}(\mathrm{CN})_{5} \mathrm{NO}\right]$. $\mathrm{H}_{2} \mathrm{O}$ shows maxima at 14840 and $21980 \mathrm{~cm}^{-1}$. In water solution, the maxima are found at $13700 \mathrm{~cm}^{-1}(\epsilon=15.2)$, $22175 \mathrm{~cm}^{-1} \quad(\epsilon=120)$ and a shoulder is observed at $30300 \mathrm{~cm}^{-1}(\epsilon=80) .^{2}$ Ignoring electron repulsion and assuming axial stabilization for the $C_{4 v}$ symmetry, Bernal and Harrison ${ }^{2}$ assigned the solution spectrum to the one electron transitions $b_{2} \rightarrow a_{1}, e \rightarrow a_{1}$, and $b_{2} \rightarrow b_{1}$, respectively. Since intra-ionic electron repulsions can hardly be ignored in evaluating the energies of the states, and since for reasons given above there may actually be axial destabilization, a more careful analysis of the visible spectrum of $\left[\mathrm{Cr}(\mathrm{CN})_{5} \mathrm{NO}^{3-}\right.$ seems desirable.

Table II lists the configurations lowest in energy for the $3 d^{5}$ ion, in terms of the crystal field and SlaterCondon parameters. On the basis of the electron resonance results $(g=1.995)$ we choose the orbital singlet ${ }^{2} B_{2}\left(e^{4} b_{2}\right)$ ground state. This ${ }^{2} B_{2}$ ground state is also expected for a negative $D t$ value, if we consider $D t$ relatively more important than $D s$. The first excited state is ${ }^{2} E(1)$, which is $3 D s-5 D t$ above the ${ }^{2} B_{2}$ state. The transition ${ }^{2} B_{2} \rightarrow{ }^{2} E(1)$ is not expected to occur at energies of the order of $14000 \mathrm{~cm}^{-1}$, so this transition will not be considered in assigning the three bands observed in the visible spectrum. Of the other excited states, the ${ }^{2} B_{1}$ and the ${ }^{2} E(2)$ should be lowest in energy, as judged by the Slater-Condon parameters. For a $B_{2}$ ground state in $C_{4 v}$, transitions to $B_{2}$ and $E$ excited states are orbitally allowed. Using this fact and also the assumption of axial destabilization, we assign the

TABLE II. Lowest spin doublets energy states for the $3 d^{5}$ electronic configuration in a strong crystal field with $C_{40}$ symmetry.

\begin{tabular}{cccc}
\hline \hline $\begin{array}{c}\text { Electron } \\
\text { con- } \\
\text { figuration }\end{array}$ & $\begin{array}{c}\text { Designa- } \\
\text { tion }\end{array}$ & $\begin{array}{c}\text { Orbital } \\
\text { energy }\end{array}$ & $\begin{array}{c}\text { Slater-Condon } \\
\text { energy } \\
\left(+10 F_{0}\right)\end{array}$ \\
\hline$e^{4} b_{2}$ & ${ }^{2} B_{2}$ & $5 \epsilon_{0}-20 D q-2 D s+15 D t$ & $-20 F_{2}-40 F_{4}$ \\
$e^{3} b_{2}{ }^{2}$ & ${ }^{2} E(1)$ & $5 \epsilon_{0}-20 D q+D s+10 D t$ & $-20 F_{2}-40 F_{4}$ \\
$e^{4} b_{1}$ & ${ }^{2} B_{1}$ & $5 \epsilon_{0}-10 D q-2 D s+15 D t$ & $-20 F_{2}-40 F_{4}$ \\
$e_{3} b_{2} b_{1}$ & ${ }^{2} E(2)$ & $5 \epsilon_{0}-10 D q+D s+10 D t$ & $-20 F_{2}-75 F_{4}$ \\
& ${ }^{2} E(3)$ & $5 \epsilon_{0}-10 D q+D s+10 D t$ & $-14 F_{2}-105 F_{4}$ \\
$e^{3} b_{2} a_{1}$ & ${ }^{2} E(4)$ & $5 \epsilon_{0}-10 D q-3 D s+4 D t$ & $-13 F_{2}-110 F_{4}$ \\
& ${ }^{2} E(5)$ & $5 \epsilon_{0}-10 D q-3 D s+4 D t$ & $-9 F_{2}-130 F_{4}$ \\
$e^{4} a_{1}$ & ${ }^{2} A_{1}$ & $5 \epsilon_{0}-10 D q-6 D s+10 D t$ & $-140 F_{4}$ \\
\hline \hline
\end{tabular}

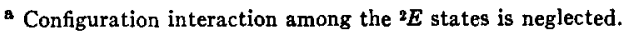


first band to the transition ${ }^{2} B_{2} \rightarrow{ }^{2} B_{1}$, and the more intense second band and the shoulder to the transitions ${ }^{2} B_{2} \rightarrow{ }^{2} E(2)$ and ${ }^{2} B_{2} \rightarrow{ }^{2} E(4)$, respectively. The separation between the ${ }^{2} B_{1}$ and the ${ }^{2} E(2)$ states is calculated to be $(3 D s-5 D t)-35 F_{4}$ while the observed energy separation between the first and second bands is approximately $7800 \mathrm{~cm}^{-1} .^{8}$ Thus if the band assignments are correct, and allowing for configuration interaction among the ${ }^{2} E$ states, we expect the ${ }^{2} B_{2} \rightarrow$ ${ }^{2} E(1)$ transition to appear in the neighborhood of $5000 \mathrm{~cm}^{-1}$.

${ }^{8}$ The $7800 \mathrm{~cm}^{-1}$ is an average of the crystal and aqueous solution values.
The assignments given in the above analysis are necessarily tentative, due to the uncertainties in the ordering of the one electron orbitals. However, there seems to be some degree of self-consistency in the assignments for both $3 d^{5}$ and $3 d^{6}$ complexes, with regard to the energies as expected from the assumption of axial compression, and with regard to the greater intensities expected for orbitally allowed transitions. Needless to say, a more detailed experimental study, especially a study of the spectra in polarized light of single crystals of the metal cyanonitrosyls, will undoubtedly allow a more unambiguous assignment of the spectral bands.

\title{
Electron Coupling of Nuclear Spins. V. Vibrational Effects in CHX Groups*
}

\author{
H. S. Gutowsky, V. D. Mochel, † AND B. G. Somers $\ddagger$ \\ Noyes Chemical Laboratory, University of Illinois, Urbana, Illinois
}

(Received September 14, 1961)

\begin{abstract}
Previous calculations of the $\mathrm{H}-\mathrm{H}$ coupling in $\mathrm{CH}_{2}$ groups assumed a static $\mathrm{H}-\mathrm{C}-\mathrm{H}$ bond angle. It is shown here that, because the predicted angular dependence of the coupling is nonlinear, the bond-bending vibrations should give an averaged value appreciably larger than that for the corresponding equilibrium, but static, angle. The contribution of excited vibrational states is very small and hence the averaged coupling constant is effectively temperature independent. There should be a small but detectable difference in the coupling constants for $\mathrm{CH}_{2}$ and $\mathrm{CHD}$ groups because of the isotopic differences in vibrational amplitude. The experimental coupling constants exhibit an apparent linear dependence upon the equilibrium $\mathrm{H}-\mathrm{C}-\mathrm{H}$ angle, and careful measurements of the isotope effects could be useful in establishing whether or not there is any explicit curvature in the angular dependence, and thus whether the observed linearity is real or due to the neglect of substituent effects in the calculations. In any case, comparison between experiment and the predicted angular dependence suggests that too low a value may have been used previously for one of the exchange integrals, $K\left(C_{1}, C_{2}\right)$ in the calculations for both the $\mathrm{CH}_{2}$ and the HCCH groups. An attempt to detect an isotopic difference between the geminal $\mathrm{H}-\mathrm{F}$ and $\mathrm{D}-\mathrm{F}$ coupling in $n-\mathrm{C}_{3} \mathrm{~F}_{7} \mathrm{H}$ and $n-\mathrm{C}_{3} \mathrm{~F}_{7} \mathrm{D}$ gave negative results.
\end{abstract}

\section{INTRODUCTION}

$\mathbf{T}$ HE dependence of nuclear spin-spin coupling constants upon molecular geometry is of considerable interest and importance. The use of valence-bond wave functions has led in several instances to calculated results in general agreement with experiment., ${ }^{1,2}$ These calculations were made for static molecules in their equilibrium configurations. However, molecular vibration, at least for light nuclei such as protons, may lead

* Acknowledgment is made to the donors of The Petroleum Research Fund, administered by the American Chemical Society, for partial support of this research. The work also was supported by the Office of Naval Research.

+ Now at Corning Glass Works, Corning, New York.

‡ Now at Phys. Lab. CIBA A. G., Basel, Switzerland.

${ }_{1} M$. Karplus, D. H. Anderson, T. C. Farrar, and H. S. Gutowsky, J. Chem. Phys. 27, 597 (1957); M. Karplus and D. H. Anderson, ibid. 30, 6 (1959).

${ }^{2}$ H. S. Gutowsky, M. Karplus, and D. M. Grant, J. Chem. Phys. 31, 1278 (1959). to averaged coupling constants appreciably different from those calculated for the static molecule. For example, it has been predicted that torsional oscillations about the $\mathrm{C}-\mathrm{C}^{\prime}$ bond in substituted ethanes should affect to an appreciable degree the proton-proton coupling in the $\mathrm{H}-\mathrm{C}-\mathrm{C}^{\prime}-\mathrm{H}^{\prime}$ group, but the symmetry of the functional form relating the coupling constant to the torsional angle, and the averaging by internal rotation, have prevented experimental verification thus far. ${ }^{3}$

In general, detection of the averaging effects requires that the vibrational amplitudes be modified. This may be accomplished, possibly without other complicating effects, either by changing the temperature of a sample or by isotopic substitution. In fact, several isotope effects upon chemical shifts have been observed in the

J. C. Schug, P. E. McMahon, and H. S. Gutowsky, J. Chem. Phys. 33, 843 (1960). 